\title{
Clinical outcome of patients treated with re-irradiation for spine or pelvic bone metastasis: A multi-institutional analysis of 98 patients
}

\author{
GEN SUZUKI $^{1}$, HIDEYA YAMAZAKI ${ }^{1}$, NORIHIRO AIBE ${ }^{1}$, KOJI MASUI ${ }^{1}$, TAKUYA KIMOTO ${ }^{1}$, \\ KOTOHA TATEKAWA ${ }^{1}$, AKINORI NAKASHIMA ${ }^{1}$, TADASHI TAKENAKA ${ }^{1}$, TAKESHI NISHIMURA ${ }^{1}$, \\ NAOMI SASAKI ${ }^{1}$, RYOONG-JIN OH ${ }^{2}$, KOJI KONISHI ${ }^{3}$, HARUUMI OKABE ${ }^{4}$, \\ SHINSUKE NAGASAWA ${ }^{5}$ and KEI YAMADA ${ }^{1}$
}

\begin{abstract}
${ }^{1}$ Department of Radiology, Graduate School of Medical Science, Kyoto Prefectural University of Medicine, Kyoto 602-8566;
${ }^{2}$ Department of Radiology, Miyakojima iGRT Clinic, Osaka 534-0021; ${ }^{3}$ Department of Radiation Oncology, Osaka Medical Center for Cancer and Cardiovascular Diseases, Osaka 537-0025; ${ }^{4}$ Department of Radiology, Uji Takeda Hospital, Uji 611-0021; ${ }^{5}$ Department of Radiology, Saiseikai Shigaken Hospital, Ritto 520-3240, Japan
\end{abstract}

Received October 11, 2016; Accepted April 3, 2017

DOI: $10.3892 /$ mco.2017.1245

\begin{abstract}
The present study aimed to describe the clinical results of re-irradiation (Re-RT) for spine or pelvic bone metastasis at the same initial irradiated area. Between April 2010 and March 2014, cases involving 98 patients with spine or pelvic bone metastasis who had undergone Re-RT at five institutions were reviewed. The clinical outcomes following Re-RT were evaluated, including overall survival (OS) and severe adverse events. The median time interval from initial radiation therapy (RT) to Re-RT was 439 days (range, 23-4,993 days), and the median duration of patient follow-up was 256 days (range, 11-2,284 days). The median biological effective dose for the Re-RT was $150 \mathrm{~Gy}_{2}$ (range, $17-240 \mathrm{~Gy}_{2} ; \alpha / \beta=2$ ). Severe late adverse events occurred in two patients who underwent three-dimensional conformal radiotherapy for lumbar spine or pelvic bone metastases, which may be associated with tumor progression. The median survival time following Re-RT was 255 days, and the actuarial OS rate at 1 year was $36 \%$. The interval between initial RT and Re-RT, and their performance statuses (PS) were significant independent prognostic factors
\end{abstract}

Correspondence to: Dr Gen Suzuki, Department of Radiology, Graduate School of Medical Science, Kyoto Prefectural University of Medicine, 465 Kajii-cho, Kawaramachi-Hirokoji, Kamigyo, Kyoto 602-8566, Japan

E-mail: gensuzu@koto.kpu-m.ac.jp

Abbreviations: BED, biologically effective dose; MST, median survival time; OS, overall survival; PS, performance status; RT, radiation therapy; Re-RT, re-irradiation; IMRT, intensity-modulated radiation therapy; SBRT, stereotactic body radiotherapy; 3D-CRT, three-dimensional conformal radiotherapy

Key words: re-irradiation, bone metastasis, prognostic factors, intensity-modulated radiation therapy, three-dimensional conformal radiotherapy for OS rates in multivariate analysis. Re-RT for spine or pelvic bone metastases is a relatively acceptable option with low risk of anticipated severe adverse events, particularly for patients with good PS following a long disease-free interval.

\section{Introduction}

Patients with bone metastasis comprise the largest group of patients receiving palliative radiation therapy (RT). RT is a widely accepted and effective way to palliate pain caused by bone metastases with few side effects (1). Local field RT has been used for patients with symptomatic bone metastasis, and a variety of dose fractionation regimens have been reported. Approximately $20 \%$ of patients require additional treatments, including re-irradiation (Re-RT) (2). Several studies have demonstrated the beneficial effect of Re-RT on pain relief (3-6); however, in cases of Re-RT, it is important to consider the tolerance dose of the normal tissue. In particular, the adverse events associated with Re-RT for bone metastases have not yet been adequately evaluated (7). In addition, the overall survival (OS) rate following Re-RT for bone metastasis remains unclear.

The present study aimed to assess the clinical results in patients with spine and pelvic bone metastases following Re-RT of the first irradiated site.

\section{Patients and methods}

A total of 103 patients with spine and pelvic bone metastases who underwent Re-RT with intensity-modulated radiation therapy (IMRT), three-dimensional conformal radiotherapy (3D-CRT), or stereotactic body radiotherapy (SBRT) were identified in five affiliated hospitals in Japan (Kyoto Prefectural University of Medicine, Kyoto; Miyakojima iGRT Clinic, Osaka; Osaka Medical Center for Cancer and Cardiovascular Diseases, Osaka; Uji Takeda Hospital, Uji; Saiseikai Shigaken Hospital, Ritto) between April 2010 and 
Table I. Patient characteristics.

\begin{tabular}{lc}
\hline Characteristics & No. of patients \\
\hline Age, years & \\
Median (range) & 61 (12-89) \\
Sex & \\
Male & 68 \\
Female & 30 \\
Performance status & \\
0-1 & 56 \\
2 & 29 \\
3 & 13 \\
Primary tumor & \\
Lung/mediastinum & 17 \\
Liver & 16 \\
Head and neck & 8 \\
Colorectal & 8 \\
Soft tissue/bone & 7 \\
Esophagus/stomach & $71-2,284)$ \\
Uterus & \\
Breast & $256(12$ \\
Others & \\
Follow-up period, days & \\
Median (range) & \\
\hline
\end{tabular}

March 2014. Of the 103 patients, five were excluded from this study as they had skipped regular follow-up visits and their data was missing. A retrospective analysis of the 98 patients who underwent Re-RT for spine and pelvic bone metastases was conducted. Re-RT was performed at the same location as the initial RT. In some of these patients, the initial RT had been performed with definitive intent or had not targeted the local relapse of spine or pelvic bone lesions. The present study included those patients in whom Re-RT was performed with curative intent (e.g., oligometastasis). All patients were informed regarding the risk of Re-RT, particularly the risk of radiation-induced myelopathy, and provided written informed consent. Patient characteristics are summarized in Table I.

The choice of 3D-CRT, IMRT or SBRT was non-randomized and primarily depended on the availability of the Linac machines or the reference for the technique by the individual physician. The time interval between RT episodes was defined as the time between the end of the initial RT and the start of Re-RT. OS was defined as the time from the end of Re-RT until the date of mortality or final follow-up of the patient. The treatment characteristics are summarized in Table II.

Acute and late adverse events following Re-RT were scored according to the Common Terminology Criteria of Adverse Events, version 4.0 (8). Acute adverse events were evaluated as those arising $<2$ months after the first day of Re-RT, and late adverse events were evaluated as those arising $\geq 2$ months after the first day of Re-RT. Newly developed neurological deficits following Re-RT were defined as late adverse events in this study. The biologically effective dose (BED) was calculated to assess the cumulative RT dose from the initial
Table II. Treatment characteristics.

\begin{tabular}{|c|c|}
\hline Characteristics & $\mathrm{N}$ \\
\hline \multicolumn{2}{|l|}{ Site of re-irradiation } \\
\hline Cervical & 17 \\
\hline Thoracic & 43 \\
\hline Lumber & 22 \\
\hline Pelvis & 15 \\
\hline \multicolumn{2}{|l|}{ Initial dose, Gy } \\
\hline Total dose, median (range) & $39(9-70)$ \\
\hline Fraction, median (range) & $13(1-32)$ \\
\hline BED2, median (range) & $73(18-174)$ \\
\hline BED10, median (range) & $48(12-101)$ \\
\hline \multicolumn{2}{|l|}{ Re-irradiated dose, Gy } \\
\hline Total dose, median (range) & $48.4(4-65)$ \\
\hline Fraction, median (range) & $10(1-25)$ \\
\hline BED2, median (range) & $150(17-240)$ \\
\hline BED10, median (range) & $75(10-89)$ \\
\hline \multicolumn{2}{|l|}{ Cumulated dose, Gy } \\
\hline BED2, median (range) & $247(57-354)$ \\
\hline BED10, median (range) & $117(29-185)$ \\
\hline \multicolumn{2}{|l|}{ Interval, days } \\
\hline Median (range) & $439(23-4,993)$ \\
\hline \multicolumn{2}{|l|}{ Radiation technique } \\
\hline IMRT & 82 \\
\hline 3D-CRT & 15 \\
\hline SBRT & 1 \\
\hline
\end{tabular}

BED2, biological effective dose in Gy for $\alpha / \beta$ value of $2 \mathrm{~Gy}$; BED10, biological effective dose in Gy for $\alpha / \beta$ value of $10 \mathrm{~Gy}$; IMRT, intensity-modulated radiotherapy; 3D-CRT, three-dimensional conformal radiotherapy; SBRT, stereotactic body radiotherapy.

RT and Re-RT, as the fractionation schemas used for the initial RT and Re-RT were varied. The BED was calculated using the following equation: $\mathrm{BED}=D \times[1+d /(\alpha / \beta)]$, as derived from the linear-quadratic model, where $D=$ the total dose and $d=$ dose per fraction. We adopted $10 \mathrm{~Gy}$ as the $\alpha / \beta$ ratio for the acute toxicities and $2 \mathrm{~Gy}$ for the late toxicities.

Statistical analysis. Data for continuous variables are presented as median (range). All statistical analyses were performed using StatView 5.0 statistical software (SAS Institute, Inc., Cary, NC, USA). Survival data were estimated by using the Kaplan-Meier method and examined for significance using the log-rank test. Cut-off values were set as the average or median value of each variable unless otherwise stated. Cox's proportional hazard model was used for the multivariate analysis. For all analyses, $\mathrm{P}<0.05$ was considered to indicate a statistically significant difference.

\section{Results}

Follow-up and survival. The median follow-up period was 256 days (range, 11-2,284 days) for all eligible patients. At 
Table III. Univariate and multivariate analysis of various potential prognostic factors for overall survival.

\begin{tabular}{|c|c|c|c|c|}
\hline \multirow[b]{2}{*}{ Characteristics } & \multicolumn{2}{|c|}{ Univariate analysis } & \multicolumn{2}{|c|}{ Multivariate analysis } \\
\hline & No. of patients & OS, 1-year rate (\%) & P-value & P-value \\
\hline \multicolumn{5}{|l|}{ Age, years } \\
\hline$<60$ & 48 & 42 & & \\
\hline$\geq 60$ & 50 & 28 & 0.23 & n.s. \\
\hline \multicolumn{5}{|l|}{ Sex } \\
\hline Male & 65 & 33 & & \\
\hline Female & 33 & 33 & 0.66 & n.s. \\
\hline \multicolumn{5}{|c|}{ Performance status } \\
\hline $0-1$ & 56 & 46 & & \\
\hline $2-3$ & 42 & 19 & 0.003 & 0.03 \\
\hline \multicolumn{5}{|l|}{ RT technique } \\
\hline IMRT/SBRT & 83 & 41 & & \\
\hline 3D-CRT & 15 & 0 & 0.08 & n.s. \\
\hline \multicolumn{5}{|l|}{ BED10 } \\
\hline$<60 \mathrm{~Gy}_{10}$ & 39 & 26 & & \\
\hline$\geq 60 \mathrm{~Gy}_{10}$ & 59 & 41 & 0.29 & n.s. \\
\hline \multicolumn{5}{|c|}{ Interval between two RT } \\
\hline$<1$-year & 44 & 14 & & \\
\hline$\geq 1$-year & 54 & 50 & $<0.0001$ & $<0.0001$ \\
\hline
\end{tabular}

the final follow-up, 41 (42\%) patients were alive and $57(58 \%)$ patients had died. The median interval from the initial RT to Re-RT was 439 days (range, 23-4,993 days). The Eastern Cooperative Oncology Group performance status (PS) of 56 patients $(57 \%)$ was 0 or 1 . Overlapping sites of the initial RT and Re-RT were as follows: Cervical spine in 16 patients, thoracic spine in 43 patients, lumbar spine in 22 patients and pelvic bone in 17 patients. The median BEDs for the initial RT and Re-RT were $73 \mathrm{~Gy}_{2}$ (range, $18-174 \mathrm{~Gy}_{2} ; \alpha / \beta=2$ ) and $150 \mathrm{~Gy}_{2}$ (range, $17-240 \mathrm{~Gy}_{2} ; \alpha / \beta=2$ ), respectively. The median cumulative BED from the initial RT and Re-RT was $247 \mathrm{~Gy}_{2}$ (range, 57-354 $\mathrm{Gy}_{2} ; \alpha / \beta=2$ ). The fractionated schemas were determined according to the condition of the patient, site of Re-RT and the discretion of the radiation oncologists at their hospital. The median survival time (MST) after Re-RT for the entire series was 255 days, and the actuarial OS rates at 1 year were $36 \%$ (Fig. 1).

Associations between survival and clinical factors. Table III presents the univariate and multivariate analysis of various potential prognostic factors for OS rates. Univariate analysis revealed that compared with patients with a PS of 2-3, patients with a PS of $0-1$ had statistically significantly higher 1-year actuarial survival rates $(\mathrm{P}=0.003 ;$ Fig. $2 \mathrm{~A})$. Similarly, when the interval between the two irradiations was $>1$ year, actuarial survival at 1 year was significantly higher $(\mathrm{P}<0.0001$; Fig. $2 \mathrm{~B})$. Additionally, $\mathrm{PS}(\mathrm{P}=0.03)$ and the interval between the two irradiations $(\mathrm{P}<0.0001)$ remained significant independent

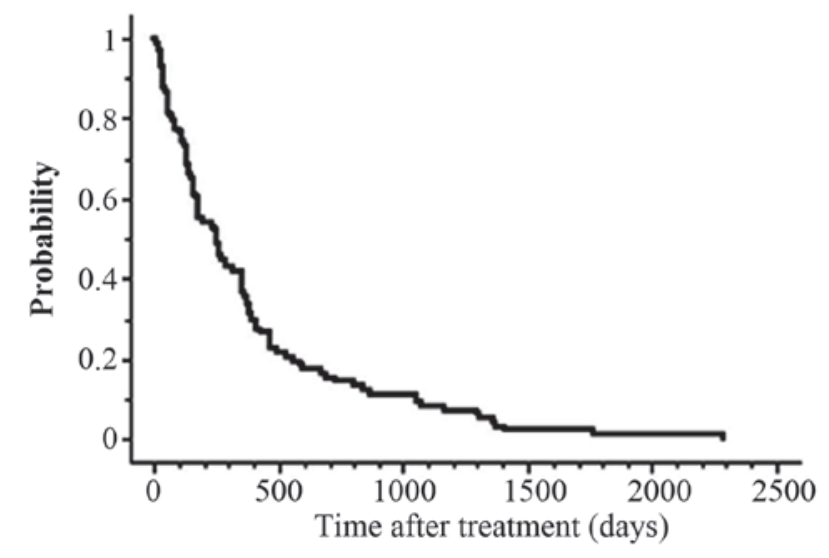

Figure 1. Overall survival curve for patients with spine or pelvic bone metastasis treated with re-irradiation.

prognostic factors for OS rates in the multivariate analysis. A BED of $60 \mathrm{~Gy}_{10}$ means a biologically equivalent dose of $50 \mathrm{~Gy}$ in 2 Gy fractions with an $\alpha / \beta$ ratio of 10 . A cut-off value of a $\mathrm{BED}$ at $60 \mathrm{~Gy}_{10}$ as the curative irradiated dose was set; however, a BED of $60 \mathrm{~Gy}_{10}$ did not have a significant effect on OS in the current study. In the multivariate analysis, PS (relative risk, 1.624; 95\% confidence interval, 1.047-2.520; $\mathrm{P}=0.03$ ) and the interval between initial RT and Re-RT (relative risk, 0.341; 95\% confidence interval, 0.217-0.536; $\mathrm{P}<0.0001)$ remained significant independent prognostic factors for OS. 

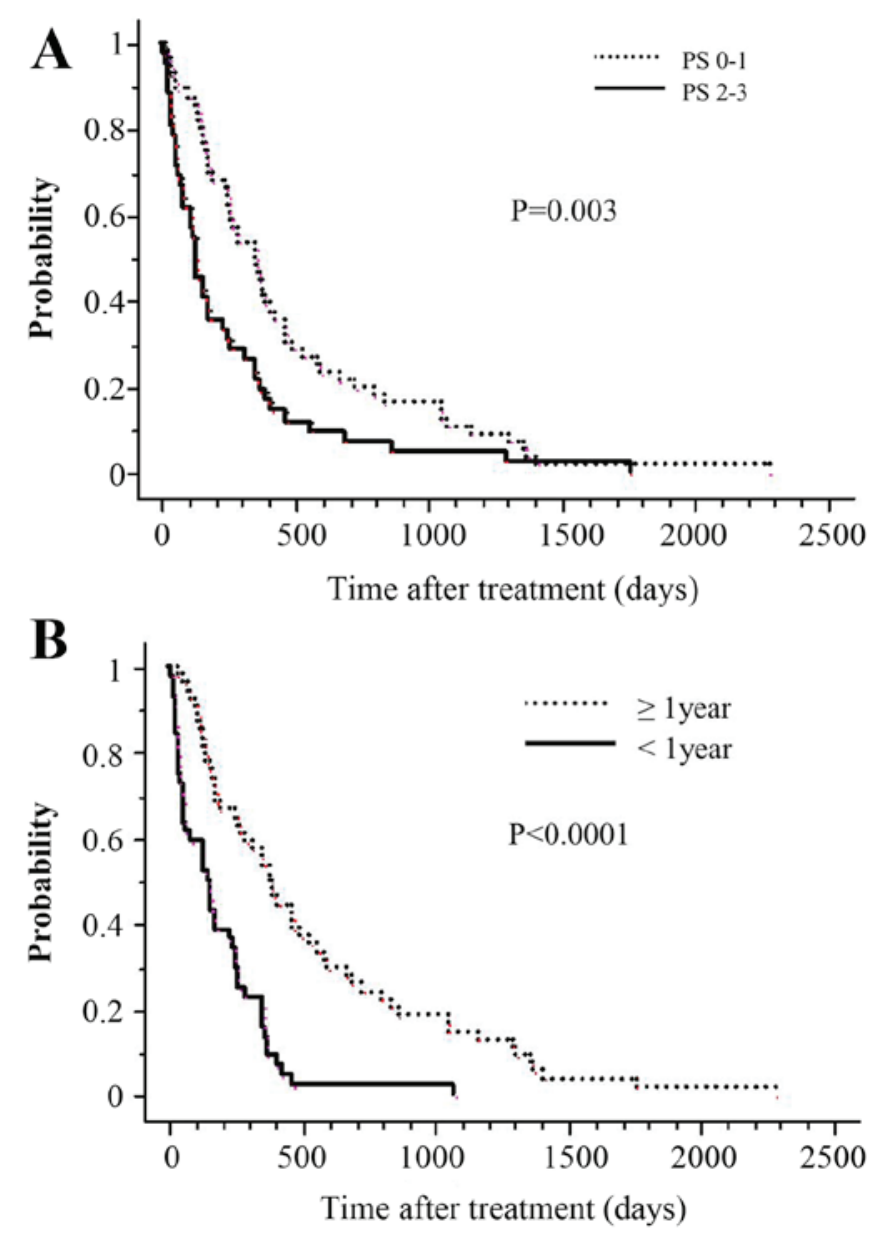

Figure 2. OS curve according to the interval between two RT administrations and PS. (A) Overall survival at 1 year for patients with PS of 2-3 before Re-RT is $19 \%$ (solid line), whereas it is $50 \%$ (dotted line) for those with PS of $0-1(\mathrm{P}=0.003)$. (B) The OS at 1 year for patients whose tumors had undergone Re-RT within 1 year of the initial RT is $18 \%$ (solid line), whereas it is $54 \%$ (dotted line) for the counterpart $(\mathrm{P}<0.0001)$. OS, overall survival; RT, radiation therapy; PS, performance status. Re-RT, re-irradiation.

Toxicities. There were no cases with acute adverse events of grade $\geq 3$. The incidence and severity of late adverse events were evaluated in the 82 patients who survived for $>2$ months after the onset of Re-RT. At the time of analysis, two cases developed grade 3-4 late adverse events, and the incidence of grade $\geq 3$ events was $2 \%(2 / 82)$.

Representative cases. Here, two representative cases in the current cohort are discussed. The first case was a 45 -year-old female with lumbar spine metastasis from cervical cancer, who received 3D-CRT with $6 \mathrm{~Gy}$ in a single fraction and suffered from grade 3 ileus, 6 months after Re-RT. The Re-RT $\mathrm{BED}$ was $24 \mathrm{~Gy}_{2}$, and the cumulative BED was $120 \mathrm{~Gy}_{2}$. The interval between initial RT and Re-RT was 6 months. The causal association between Re-RT and the occurrence of ileus was unclear, as this case also had peritoneal dissemination. The second case was a 67-year-old male with a bulky sacral metastasis from hepatocellular carcinoma, who received 3D-CRT of 20 Gy in four fractions once daily. He suffered from grade 4 injury to the cauda equina 2 months after the Re-RT. The Re-RT BED was $70 \mathrm{~Gy}_{2}$, and the cumulative BED was $168 \mathrm{~Gy}_{2}$. The interval between the initial RT and Re-RT was 7 months. None of the cases treated with IMRT experienced grade $\geq 3$ toxicity.

\section{Discussion}

The current study performed a retrospective multi-institutional analysis of patients treated with Re-RT for metastatic disease in the spine or pelvic bone. The results demonstrated that Re-RT performed for spine or pelvic bone metastases was associated with a comparatively acceptable rate of severe adverse events. The most important adverse effect of Re-RT was radiation-induced myelopathy or radiation-induced injury to the cauda equina. In the present study, one patient treated with 3D-CRT was suspected to have injury to the cauda equina; however, it is not completely certain that this particular case developed radiation-induced injury to the cauda equinal as there are no clinical features that are able to differentiate between radiation-induced injury and compressive injury with certainty. Furthermore, in this case, follow-up studies (e.g., MRI, PET or CT) were not able to be conducted due to the patient's extremely poor general condition.

Regarding late toxicities associated with Re-RT for spine metastases, the criteria of Nieder et al $(9,10)$ prove to be useful indices for reference in evaluating the safety of Re-RT. These indices suggested that the risk of radiation myelopathy caused by Re-RT was low when the cumulative BED from the initial $\mathrm{RT}$ and Re-RT was $<135.5 \mathrm{~Gy}_{2}$ and the interval between the initial RT and Re-RT was not $<6$ months. The risk was low when the BED of each course was not $>98 \mathrm{~Gy}_{2}(9,10)$. Although the patient was suspected to have radiation-induced injury to the cauda equina, the cumulative BED2 for both treatments was $124 \mathrm{~Gy}_{2}$, and the interval between the initial RT and Re-RT was 6 months. The initial BED was $75 \mathrm{~Gy}_{2}$, and the second was $49 \mathrm{~Gy}_{2}$. The onset of signs and symptoms was seen 2 months after Re-RT. During the follow-up, no severe toxicities, including radiation-induced myelopathy, occurred for any of the patients treated with IMRT; therefore, it was inferred that the cumulative doses to the spinal cord and cauda equina were within the permissible range as given in published reports $(9,10)$. IMRT can be relatively more flexible in designing a dose distribution fitting a lesion with a concave shape than 3D-CRT. This distinction may be useful in the irradiation of spine or pelvic bone metastases, particularly for Re-RT. Kawashiro et al (11) reported clinical IMRT results of Re-RT for spinal metastasis. They concluded that spinal metastasis Re-RT using IMRT was safe and that pain relief and paresis improvement and/or prevention may be expected along with a reduced risk of radiation-induced toxicity, especially in the spinal cord (11). In addition, Jereczek-Fossa et al (12) reported that the low toxicity of Re-RT with IMRT or SBRT should allow for delivery of higher doses and lead to improvement in Re-RT outcomes.

The present study found a favorable 1-year OS rate of $36 \%$, and the MST following Re-RT for the entire series was 255 days (range, 7-2,285 days). Hayashi et al (3) found that the MST after Re-RT was of 4 months. Similarly, Hernanz et al (13) identified that the MST following Re-RT was of 3 months. Additionally, they noted that the survival was significantly longer when the interval between initial RT and Re-RT was $>1$ year (13). It must be noted that the results of the 
present study are consistent with those of this previous report. Other factors, such as Karnofsky Performance Status prior to treatment or the administered BED were not significantly associated with survival in their study (13). In the present study, multivariate analysis revealed that not only the intervals between initial RT and Re-RT but also PS at the time of Re-RT were observed as statistically significant prognostic factors. The finding of PS as a prognostic factor in the current study is consistent with certain previous reports $(14,15)$.

Regarding pain improvement, the present study was not able to quantitatively determine the degree of improvement, as the pain was not objectively recorded prior to and following Re-RT in the majority of the patients. Furthermore, details of medication were not reliably collected to determine whether the subjectively documented pain response was confounded by the use of analgesics and varying supportive care. For the same reason, mild toxicities of grade $\leq 2$ were also difficult to evaluate in the current study. These issues are considered to be limitations of the study. Documentation of severe toxicities is more reliable despite the retrospective nature of the study given the permanent nature of the event. Most importantly, we experienced two cases with severe late toxicities of grade $\geq 3$ : One case of grade 3 ileus and one case of radiation-induced injury to the cauda equina, both of which were treated with 3D-CRT.

In recent years, as a direct result of progress in anticancer therapy, the survival rate of patients with distant metastatic disease has been improving. With this increasing rate of survival, there is an increasing requirement for Re-RT in these patients. Wong et al (16) reported that latent times for myelopathy following a single course of treatment (mean, 18.5 months; range, 7-57 months) were significantly longer than those following Re-RT (mean, 11.4 months; range, 4-25 months). Re-RT using IMRT or SBRT, which is able to achieve a greater dose prescription than $3 \mathrm{D}-\mathrm{CRT}$, for metastatic spine or pelvic bone may provide the possibility of lasting tumor control, as well as prevention of radiation myelopathy and radiationinduced injury to the cauda equina.

In conclusion, the present data suggest that Re-RT using IMRT or SBRT is safer than 3D-CRT. In particular, patients with a long interval from initial radiation and good PS (0-1) may survive long enough to benefit from local intensive RT, such as IMRT or SBRT.

\section{Acknowledgements}

The authors would like to thank Enago (www.enago.jp) for the English language review.

\section{References}

1. Lutz S, Berk L, Chang E, Chow E, Hahn C, Hoskin P, Howell D, Konski A, Kachnic L, Lo S, et al; American Society for Radiation Oncology (ASTRO): Palliative radiotherapy for bone metastases: an ASTRO evidence-based guideline. Int J Radiat Oncol Biol Phys 79: 965-976, 2011.
2. Huisman M, van den Bosch MA, Wijlemans JW, van Vulpen M, van der Linden YM and Verkooijen HM: Effectiveness of reirradiation for painful bone metastases: a systematic review and meta-analysis. Int J Radiat Oncol Biol Phys 84: 8-14, 2012.

3. Hayashi S, Hoshi H and Iida T: Reirradiation with local-field radiotherapy for painful bone metastases. Radiat Med 20: 231-236, 2002.

4. Jeremic B, Shibamoto Y and Igrutinovic I: Single 4 Gy re-irradiation for painful bone metastasis following single fraction radiotherapy. Radiother Oncol 52: 123-127, 1999.

5. Mithal NP, Needham PR and Hoskin PJ: Retreatment with radiotherapy for painful bone metastases. Int J Radiat Oncol Biol Phys 29: 1011-1014, 1994.

6. van der Linden YM, Lok JJ, Steenland E, Martijn H, van Houwelingen H, Marijnen CA and Leer JW; Dutch Bone Metastasis Study Group: Single fraction radiotherapy is efficacious: a further analysis of the Dutch Bone Metastasis Study controlling for the influence of retreatment. Int J Radiat Oncol Biol Phys 59: 528-537, 2004.

7. Wong E, Hoskin P, Bedard G, Poon M, Zeng L, Lam H, Vulpe H, Tsao M, Pulenzas N and Chow E: Re-irradiation for painful bone metastases - a systematic review. Radiother Oncol 110: 61-70, 2014.

8. National Cancer Institute: Common Terminology Criteria for Adverse Events (CTCAE) version 4.0. https://ctep.cancer. gov/protocoldevelopment/electronic_applications/ctc.htm\#ctc_40. Accessed January 8, 2016.

9. Nieder C, Grosu AL, Andratschke NH and Molls M: Proposal of human spinal cord reirradiation dose based on collection of data from 40 patients. Int J Radiat Oncol Biol Phys 61: 851-855, 2005.

10. Nieder C, Grosu AL, Andratschke NH and Molls M: Update of human spinal cord reirradiation tolerance based on additional data from 38 patients. Int J Radiat Oncol Biol Phys 66: 1446-1449, 2006.

11. Kawashiro S, Harada H, Katagiri H, Asakura H, Ogawa H, Onoe T, Sumita K, Murayama S, Murata H, Nemoto K, et al: Reirradiation of spinal metastases with intensity-modulated radiation therapy: an analysis of 23 patients. J Radiat Res (Tokyo) 57: 150-156, 2016.

12. Jereczek-Fossa BA, Kowalczyk A, D'Onofrio A, Catalano G, Garibaldi C, Boboc G, Vitolo V, Leonardi MC, Cambria R and Orecchia R: Three-dimensional conformal or stereotactic reirradiation of recurrent, metastatic or new primary tumors. Analysis of 108 patients. Strahlenther Onkol 184: 36-40, 2008.

13. Hernanz R, Montero A, Fernandez-Lizarbe E, Polo A and Ramos A: Retreatment with radiotherapy for symptomatic bone, brain or visceral metastases. Clin Transl Oncol 15: 72-78, 2013.

14. Hashmi A, Guckenberger M, Kersh R, Gerszten PC, Mantel F, Grills IS, Flickinger JC, Shin JH, Fahim DK, Winey B, et al: Re-irradiation stereotactic body radiotherapy for spinal metastases: a multi-institutional outcome analysis. J Neurosurg Spine 25: 646-653, 2016.

15. Damast S, Wright J, Bilsky M, Hsu M, Zhang Z, Lovelock M, Cox B, Zatcky J and Yamada Y: Impact of dose on local failure rates after image-guided reirradiation of recurrent paraspinal metastases. Int J Radiat Oncol Biol Phys 81: 819-826, 2011.

16. Wong CS, Van Dyk J, Milosevic M and Laperriere NJ: Radiation myelopathy following single courses of radiotherapy and retreatment. Int J Radiat Oncol Biol Phys 30: 575-581, 1994. 